\title{
Effects of Gene Expression in the United Universe
}

\section{Stupar $\mathbf{M}^{*}$ and Stefanovic S}

Institute for Nuclear Science Vinca, Beograd, Serbia

\section{Description}

Within this title, the pivotal role is assigned to the four fundamental forces in the Universe: gravity, electromagnetism, and weak and strong nuclear forces, from one side, and an uncertainty biological principle. This brief overview is a derivative of upcoming theory of the unification of Physics and Biology into a Super Theory of Everything.

In the last few decades, string theory has emerged as the most promising candidate for a microscopic theory of gravity. Strings are one-dimensional extend objects of 10-35 $\mathrm{m}$ in length. They can be seen as a messenger particle with enormous tension, of about 10 to 42 kilograms. Physicists say if string theory is correct, then entire world is made of strings. Wrong.

Physicist managed to pulverize the electron into three composite nebular parts: spinon, holon, and orbiton. In the photon double cascade, from a single photon, three were obtained. Neutrino exists also in three forms - tau, electron, and muon. It can be that all astroparticles spend a little time as combination of all other particles, i.e. as united ones, long enough to remember each other's. It speaks in favor that astro-particles know each other's, that they have awareness of other particles. Something is there whose property is to unify all the Universe. This fits into the role of dark matter and the transmission of information in the biological systems.

Of all four physical forces, none of them can explain what's alive is. Which quality makes the difference between these forces and living organisms? The human beings can explain physical structures, but other way round explanations do not exist. In living organisms, all of the puzzles in the cells are based on the biochemical reactions. In these pathways all participants are materialistic, realistic. DNA as a matter that is visible under the electronic microscope, is transcribed to the RNA, which is in its own turn visible under electronic microscope. The finale product of gene expression is protein, also the major physical entities. But, what is that in biochemistry that makes biology? The answer to this question can be found in the way the brain functions. The basic role of the brain is to think, to produce ideas, plans. Analyzing the expression of the genes in the brain, looking for biochemical processes involved in the production of the thoughts, ideas, none of it has been found. The conclusion is clear, our brain, and even all bio-kingdom, does not produce thoughts, it means that we do not think!!! Who then thinks instead? This conclusion is fine, alright, because thoughts is not a physical phenomenon, it has no mass, it does not turn in gravitational field, there is no frequency, in one word speaking there is nothing in common with physical entities, in our koino-observable Universe.

As billions of nerve cells interweave in the brain, the same does another kind of matter interweave in the Cosmos. The dark matter communicates in all parts of the Universe, as does microRNA (miRNA) in the human body, transmitting information of greatest importance; bearing in "mind" the emerging evidence that extracellular vesicles (EV) associated miRNA play important role in cancer. Tumor cells secrete EV containing miRNA that serve as "communication shuttles"” between cells. In this way the ancient RNA WORLD roaming across human body, transmitting memory through the evolution. The same role has dark matter protecting the galaxy from collapse and disintegration. So that, in material world there are three types of matter: koino matter, the dark matter, and energy (including biochemical products). In this way there are two properties of Universe; matter and thoughtful quality.

The proof for this claim is two initial experimental results that confirm existence of million (thoughtful quality). In the book "Progress in String Theory Research" the chapter four under the title: "DNA, water, and strings united by mislion, results in life", explains this phenomenon (1). If we want to unite all physical and thought processes in a single event, we need to go a step deeper, to break barrier that prevents us from relaxing in the searching of most sensitive mysteries in the Cosmos. To unite these two properties of the Universe-thoughts processes and matter, they must be united in one force searching for the third common denominator. Without answering to this question, none of the theory of the origin of the Universe, as well as the purpose for its existence, is not valid and complete. It seems that we'll wait for a new upcoming ribosomal organelle.

In addition to the two already existing organelles, mitochondrial and chloroplast, should be formed and third ones (realizing that the nucleus is not an organelle in the strict sense). The third one is a ribosomal organelle (2). The purpose of existence of the organelles, which will be subject of new project, and particular ribosomal ones, would be the basis for an attempt to find out the secrets of the common denominator. During the evolutionary course, all the genes expressing ribosomal proteins and ribosomal RNAs (rRNA) genes are transferred from mitochondria to nucleus. Mitochondrial DNA contain two ribosomal RNAs genes (12S and 16S), not yet transferred to the nucleus. These two, should be, soon or later, landed in the nucleus. In the nucleus everything has been already prepared, there are chromosomally encoded all of the ribosomal protein genes, nucleolar organizers, nucleolus, and $45 \mathrm{rDNA}$, waiting for $12 \mathrm{~S}$ rRNA and $16 \mathrm{~S}$ rRNA genes to joining them to form the third DNA containing organelle, ribosomal compartment. What is the reason for this major ever moment in the existence of life in the whole Universe? Advent of the ribosomal organelle will have enormous impact on reproductive characteristics and on intellectual (thoughts, abstract and complex ideas, imagination, dreams, spirit) activity of the brain. This is the answer to the question: whether human still evolving? [1-5].

\section{References}

1. Davis FP (2015) Progress in String Theory Research. Nova Sience Publishers, United Kingdom.

2. Stupar M, Stefanovic S, Vidovic $\vee(2008)$ Strings, water and DNA united by

*Corresponding author: Dr. Stupar Milanko, Institute for Nuclear Science Vinca, Beograd, Serbia, Tel: +381 11 3408101; E-mail: milanko.stupar@laposte.net

Received February 05, 2018; Accepted February 09 2018; Published February 12, 2018

Citation: Stupar M, Stefanovic S (2018) Effects of Gene Expression in the United Universe. J Mol Genet Med 12: 329 doi:10.4172/1747-0862.1000329

Copyright: $\odot 2018$ Stupar M, et al. This is an open-access article distributed under the terms of the Creative Commons Attribution License, which permits unrestricted use, distribution, and reproduction in any medium, provided the original author and source are credited 
mislion results in life. In: Progress in String Theory Research. Nova Sience Publishers, United Kingdom.

3. Stupar M, Stefanovic S, Vidovic V (2015) Ribosomal DNA intercellular journey and the new upcoming ribosomal organelle. Iranian J of Sci Technol 39: 267-272.
4. Gray MW (1988) Organelle origins and ribosomal RNA. Biochem Cell Biol 66: 325-348.

5. Gerbi SA (1985) Evolution of Ribosomal DNA. Mol Evol Genet 1: 419-517. 